\title{
The effects of competition (intra-specific and inter- specific) and predation on the distribution and abundance of guppy fish (Poecilia reticulata)
}

\begin{abstract}
Competition and predation are among the major evolutionarily and ecological forces which play imperative role to determine the distribution, abundance, and population dynamics of species in a biotic community. The objective of this study was to test the effects of competition (i.e. intra-specific and interspecific) and predation on the distribution, abundance, and fitness of guppy fish. The data were collected in laboratory experiments where guppies were kept in homogenous aquarium with different densities in each partition under the influence of goldfish as competitors and predators. The experiment was conducted in three different stages: only guppies were kept in the aquarium, goldfish were used as competitors, and different densities of predatory fish (i.e. goldfish) were kept in both sides of the aquarium. Regression, isocline, and isodar methods were employed to analyze the data to measure the impacts of competition and predation on the distribution, abundance, and fitness of guppies and later to determine which factor is more influential over the other. The results revealed that both competition and predation were shown to be important evolutionarily and ecological forces to affect the distribution, abundance, and fitness of guppies. Competition coefficient was affected by the densities of both guppies and goldfish; however, predation coefficient was affected only by the density of goldfish. The results of the coefficients of competition and predation suggested that the effect was density-dependent. Generally, predation had the strongest effect on the distribution, abundance, and fitness of guppies followed by the effects of interspecific and intra-specific competitions, respectively.
\end{abstract}

Keywords: aquarium, biotic community, coefficients, density-dependent, fitness, goldfish
Volume 3 Issue 5 - 2018

\author{
Solomon Ayele Tadesse \\ Department of Natural Resources Management, College of \\ Agriculture and Natural Resource Sciences, Ethiopia \\ Correspondence: Solomon Ayele Tadesse, Department of \\ Natural Resources Management, College of Agriculture and \\ Natural Resource Sciences, Debre Berhan University, PO Box \\ 445, Debre Berhan, Ethiopia, Tel +25 I I II68 I544 0 (office), \\ +25I946703660 (mobile), Fax +25II II68 I206 5, \\ Email solomon.ayele1972@gmail.com
}

Received: March 26, 2018 | Published: September 28, 2018

\section{Introduction}

Organisms select habitats to acquire and secure their living essentials in various ways. Naturally different species of organisms have their own organizational structures in a biotic community ${ }^{1}$ to exploit the existing recourses. ${ }^{2-4}$ This ultimately leads to different evolutionarily and ecological complexities, interactions, and interdependencies among different species in a biotic community. For example, species have different niche breadths which are affected by the variability of the environment, and the relationship with other species in the habitat, ${ }^{5,6}$ and therefore have different selectivity and preference for habitats. ${ }^{5}$ Competition (i.e. intra-specific and interspecific), predation, mutualism, and commensalisms are the major ecological interactions and interdependencies which influence the structure and the function of ecosystems. ${ }^{2,6,7}$ Species exhibit different habitat preferences and thus are patchy in their distribution, diversity, and hence the system is dynamic. ${ }^{8}$ Species distribution and abundance depends on different factors. For example, competitively mediated habitat selection creates the disturbing paradox that the competition responsible for habitat segregation lurks as the 'ghost of competition past', ${ }^{9-11}$ which is invisible at the point of equilibrium.

Understanding the process of habitat selection is of fundamental importance in determining the extent and fate of population interactions and distributions. ${ }^{8,12}$ Ecologists have long sought to link behavior, population dynamics, and the rules of ecological community organization by using optimal foraging theories. ${ }^{13-16}$ Densitydependent habitat selection theory applies to all species independent of their life-history characteristics or the number of habitats available in the landscape. ${ }^{12,17-21}$ Most studies on habitat selection dealt with how environmental variable, ${ }^{22}$ intra-specific and inter-specific competitions, ${ }^{5,10}$ and predation ${ }^{7}$ affect habitat selection and preference of species in their natural habitats. Many ecologists have proved that the preference for one habitat among the many implies that the species has higher fitness in that habitat. ${ }^{23-25}$

A central challenge in ecology is to develop models that faithfully capture those important mechanistic details of natural systems that are required to make reliable predictions about population dynamics. ${ }^{26}$ Many theories that deal with the different factors of organisms' habitat selection are developed based on the assumption of IFD (Ideal Free Distribution). ${ }^{23}$ This is because IFD provides the basis for understanding how individuals should distribute themselves among different habitats in response to habitat quality and population density. ${ }^{18,19,23}$ The IFD assumes that foragers can change habitats without cost. Individuals choose the habitat that offers the highest fitness, and individuals can enter a habitat on an equal basis with those already there. Moreover, the IFD assumes that suitability or fitness of individual in a habitat declines with the habitat's population density and species have full knowledge of their habitats, so they react in 
response to changes in densities and resources. ${ }^{23}$ When there is more than one habitat, at low densities, the most preferred habitat would be taken first. Then, as the density increases, the fitness of the species decreases to a point where it is better to move into the second best habitat in order to increase its fitness. ${ }^{18,19,24}$

In the present study, it is sought imperative to consider the combined effects of different environmental variables, ecological complexities, interdependencies, and interactions which significantly affect the mechanisms of habitat selection in guppies under the influence of competition (i.e. intra-specific and interspecific) and predation. Thus, it is better to evaluate separately the impacts of competition and predation to measure and compare their separate and combined effects on the distribution, abundance, and fitness of guppies. Moreover, measuring the impact of intra-specific competition when competitors or predators are present and the effect of competitors and predators densities on their coefficients is imperative to evaluate and understand whether all those factors equally affect the distribution, abundance, and fitness of guppies or there is some significant difference among those factors in affecting the distribution, abundance, and fitness. Thus, the objective of the study was to test the effects of competition (i.e. intra-specific and inter-specific) and predation on the distribution, abundance, and fitness of guppies in an experimental aquarium under laboratory condition.

\section{Conceptual research background}

\section{The effect of competition and predation on the distribution, abundance, and fitness of guppies}

Competition and predation are pervasive evolutionarily and ecological forces which play imperative role to determine the distribution, abundance, habitat selection, behavior, fitness, and population dynamics of different species in a biotic community., $4,12-15$ Thus, it is better to separately discuss how competition and predation affect the distribution, abundance, and fitness of organisms in a biotic community.

Competition: is one of the evolutionarily and ecological complexities which affects the real ecological niches of species in a biotic community. ${ }^{4}$ Competition is often seen among individuals of the same or different species which use similar habitat resources in a similar way. ${ }^{5}$ For better understanding of this competition type (i.e. intraspecific and interspecific), I briefly discuss each type of competition as followed.

Intra-specific competition: It is a kind of competition in which individuals of the same species may compete for the same resources due to either the scarcity of common habitat resources or due to higher density. ${ }^{3-5}$ For example, according to the IFD assumptions, density was demonstrated to have a paramount effect on the habitat selectivity and resource utilization by individuals of a species. ${ }^{19-21,25}$ Several ecological studies which were conducted on the habitat selections of different species have clearly shown that habitat selection by individuals of a species is affected by their own densities. ${ }^{19-21}$ For example, Abramsky et al. ${ }^{12}$ developed a dispersion method to demonstrate how distribution and habitat selection in desert rodents are affected by their own densities. Accordingly, Abramsky et al. ${ }^{12}$ studied desert rodent species whether they are more habitat selective, density-dependent or randomly distributed between habitats. Thus, the geometrical method that was developed by Rosenzweig and Abramsky et al. ${ }^{12}$ was able to detect changes in selectivity level with changes in species densities. Moreover, isodar theory ${ }^{19-21}$ and isocline methodwere able to measure the effect of intra-specific competition while competitors or predators are present. Thus, based on those theories and methods, this study was conducted to test how density and resource availability (i.e. food quantity) affect the distribution, abundance, and fitness of guppies. ${ }^{27}$

Inter-specific competition: Various ecological researchers proved in their research works that inter-specific competition is one of the natural selective forces that influence the ecological niche breadth, distribution, abundance, habitat selection, and resource utilization of various species..$^{3-5,12-14,28}$ For example, a number of theories have been tested to measure the effects of inter-specific competition in the field, and different methods were employed to compute the effect of interspecific competition. ${ }^{5,7,17-19}$ Moreover, methods such as specific indices of niche overlap, ${ }^{22}$ are based on the assumption that competition coefficient between two species is constant and bi-directional. Other methods such as Mac-Arthur \& Levins ${ }^{6}$ measure of niche overlap are based on different effect of each species on the other, but there is a difficulty in measuring those effects in nature, since it is difficult to separate the impacts of different species and environmental variables. ${ }^{7}$ Competition coefficient is one of the important variables which help explain the degree of inter-specific competition between individuals of different species in a biotic community. ${ }^{5}$

However, Crowell \& $\mathrm{Pimm}^{29}$ demonstrated that competition coefficient is measurable among different species only in a homogenous environment. Isoclines are also drawn from a set of census data to measure the effects of inter-specific competition among different competing species in a biotic community., ${ }^{5,70}$ For example; Vandermeer ${ }^{31}$ demonstrated that once the slope of the isocline is known, it is easy to compute the competition coefficients between two competing species for the same habitat resource in a biotic community. Multiple regression method is also another suggested option to solve the problem of environmental heterogeneity in field experiments to compute inter-specific competition coefficients between two competing species. ${ }^{5,29,30}$ All the aforementioned methods of competition coefficient determining techniques used to and have been still using species densities and variability of food resource to measure the inter-specific competition between ecologically competing species for similar habitat resources.

In the present study, guppies and goldfish were the two competing species for the same food resource. Thus, the interspecific competition coefficients between these two fish species were computed. A regression method was used to test whether competition coefficients between the two fish species are affected by density and food availability. ${ }^{5,17,30}$ Rosenzweig9 has theoretically shown that habitat selection models promote the possibility of coexistence by changing the shape of the species zero growth isoclines from linear to non-linear. This study also looked into the effect of inter-specific competition on the distribution, abundance, and fitness of guppies in an experimental aquarium

Predation: Predators directly affect their prey by killing and consuming them. However, this is not the only way they can affect them. Non-lethal effects, such as fear and apprehension generated from the possibility of being attacked may be enough to change prey behavior. ${ }^{5,26,31-36}$ Non-lethal effects affect the fitness of prey individuals because the prey's options are constrained and the behavioral response may be costly. ${ }^{26,35}$ and even physiologically stressful when it is at its extreme case. ${ }^{36}$ For example, previous studies on invertebrates ${ }^{37-39}$ and 
amphibians ${ }^{40}$ have shown that non-lethal effects may be larger than lethal effects in determining the habitat section, patch use, foraging behavior, morphology, survival and reproductive rates, activity density, time budgets, distribution, and abundance of animals over a range of trophic levels. ${ }^{26,36}$

Due to the potentially high costs of avoiding predation and the tendency for large portions of populations to respond to risk of predation, the risk effects of predators can have impacts on prey species and communities that may even surpass those of direct predation, ${ }^{41}$ because effect of risks can exist even when direct rate of predation is zero. ${ }^{42}$ So, to reduce the negative impacts of predation risk, prey may avoid certain habitats, spend more time being vigilant and less time feeding, feed in larger groups, use apprehension or restrict their feeding to certain times..$^{26,42,43}$ Ultimately, risk-sensitive behavior resulted from non-lethal causes may affect the foraging behavior, distribution, abundance, habitat selection, and patch use by prey species.

Lima \& Dill ${ }^{28}$ showed that predation has long been implicated as a major selective force in the evolution of several morphological and behavioral characteristic of prey species. Predation could affect the fitness of individuals of a species in a biotic community. Thus, prey species need to protect themselves from risk of predation which adds costs on the foraging efficiency of the forager. ${ }^{7,15,44,46}$ For example, Abramsky et al..$^{5}$ and Kotler et al. ${ }^{7}$ demonstrated that predation was stronger than competition in affecting the activity levels of two rodent species (i.e. Gerbillus pyramidum and Gerbillus allenbyi) in the sandy habitats of the Negev Desert, Israel. In this study, the impact of predation on the distribution, abundance, and fitness of guppies was computed and later compared with the impacts of intra-specific and inter-specific competitions. This helped to identify the strongest force which significantly affects the distribution, abundance, and fitness of guppies in an experimental aquarium under laboratory condition.

\section{Materials and methods}

\section{Study species}

\section{Guppy}

The guppy (Poecilia reticulata), also known as rainbow fish, is one of the world's most widely distributed tropical fish. ${ }^{47}$ It is also one of the most popular freshwater aquarium fish species. Although not typically found there, guppies also have tolerance to brackish water and have colonized some brackish habitats. ${ }^{48}$ However, they tend to be more abundant in smaller streams and pools than in large, deep or fast-flowing rivers. ${ }^{49}$ Guppies, whose natural range is in northeast South America, were introduced to many habitats and are now found all over the world. ${ }^{47,49}$ They are highly adaptable and thrive in many different environmental and ecological conditions. ${ }^{47}$ Wild guppies generally feed on a variety of food sources, including benthic algae and aquatic insect larvae. ${ }^{50}$ Guppies have many predators, such as large fish (e.g. goldfish) and birds, in their natural habitats..$^{50,51}$ Like many fish species, guppies often school together to avoid predation. Schooling is more favored by evolution in populations of guppies under high predation pressure, exerted either by predator type or predator density. Coloration of guppies also evolves differentially in response to predation. ${ }^{51}$ Guppies are used as a model organism in the field of ecology, evolution, and behavioral studies. ${ }^{47}$

Goldfish: Goldfish (Carassius auratus) is a freshwater fish species. It is one of the most commonly kept aquarium fish. The goldfish is native to East Asia. ${ }^{52}$ It was first selectively bred in China more than a thousand years ago, and several distinct breeds have since been developed. Goldfish breeds vary greatly in size, body shape, fin configuration, and coloration. ${ }^{53}$ Goldfish are gregarious, displaying schooling behavior, as well as displaying territorial behavior while feeding. ${ }^{54}$ They are a generalist species with varied feeding and breeding behaviors that contribute to their success. Goldfish are opportunistic feeders and do not stop eating on their own accord. ${ }^{52-54}$

\section{Experimental procedures}

The data were collected from laboratory experiments where guppies were kept in homogenous aquarium with different densities. The aquarium was divided into two partitions with a string net as a barrier. The dimension of each partition measures $50 \mathrm{~cm} \times 50 \mathrm{~cm}$. The barriers made up of string nets were big enough to allow the free movement and passage of the guppies between the two partitions of the aquarium, but bigger fish (i.e. goldfish) could not. First, the guppies were trained to recognize knocks in the aquarium glass as a preliminary sign for food provision. Knocking was simultaneously done in both sides of the aquarium before feeding, and guppies were acquainted for such a response before the actual data collection was started.

\section{Experimental data collection}

The experiment was conducted for 12 weeks in three different stages. The experiment for each stage lasted within four weeks. The data were collected as followed.

Only guppies were in the aquarium: Equal amount of food pellets was added to both sides of the aquarium. This was done to test whether guppies utilize the provisioned food pellets in both sides similarly. Food pellet was given to the guppies in the amount of 0.5 pellets per fish in the beginning, but one pellet per fish was provided later. Each pellet weighed $1 \mathrm{gm}$. The number of guppies in each side of the aquarium was counted to measure their distribution and density.

Goldfish were used as competitors and kept in one side of the aquarium in two different densities: Goldfish were unable to pass through the small string nets so that they were not allowed to move to the other side of the aquarium. Food was added to both sides of the aquarium in equal quantity. Finally, the data were collected by counting the number of guppies and goldfish in both sides of the aquarium. This activity is important to measure the effect of the competitors (i.e. goldfish) on the distribution, abundance, and competitive fitness of guppies. Food pellet was given to the fish (i.e. guppies and goldfish) in an amount of 1 pellet per fish including the competitor goldfish.

Different densities of predatory fish were kept in both sides of the aquarium: The goldfish were unable to pass from one side of the aquarium to the other. Food pellet was added to both sides of the aquarium in equal proportion. The data were collected by counting the number of guppies on both sides of the aquarium. Moreover, the number of the predatory fish (i.e. goldfish) was also counted. This activity was important to measure the effect of predators on the distribution, abundance, and fitness of guppies in each partition of the experimental aquarium.

\section{Data analyses}

First, I used one way ANOVA to analyze and measure the effect of time on the distribution and abundance of guppies where competitors (e.g. goldfish) were present in one side of the aquarium. 
The isodar method: According to Morris, ${ }^{18,19}$ the isodar method can be employed to measure the fitness of a single species so that it can be computed with a simple linear regression technique by regressing the densities of the study species in two adjacent habitats. Thus, for species A when alone, the isodar is defined by the following equation:

$$
\mathrm{NA} 1=\mathrm{C}-\mathrm{b} \text { NA2 }
$$

Where,

NA1 and NA2 = density of species A in habitat 1 and habitat 2 , respectively.

$\mathrm{C}=$ Intercept that reflects the quantitative difference between the two habitats.

$\mathrm{B}=$ slope that shows the qualitative difference of habitat 2 compared with habitat 1 .

However, if both habitats are identical; then, $\mathrm{C}=0$ and $\mathrm{b}=1$.

Effects of inter-specific competition and predation: Competition and predation coefficients were computed based on the method developed by Abramsky et al..$^{5}$ Accordingly, the different activity densities of guppies were regressed to examine the preference of the guppies and to draw the isoclines. Hence, the slopes of the isoclines were measured at different densities. It helped determine competition coefficients and evaluate its relation with the change in densities. Moreover, different densities of guppies and the fixed number of competitors (i.e. goldfish) in one side of the aquarium were regressed. The different densities of guppies were also regressed against the densities of the predatory fish (i.e. goldfish).

I was able to draw one isocline from the average densities of guppies and competitors and/or predators in each replicate of the collected data from the aquarium experiment. This isocline was a line between two points on a graph, where guppies' density was in the Y axis but competitor/predator density was in the $\mathrm{X}$ axis. The different densities of the guppies allowed me to know the effect of guppies' density on competition coefficients. Moreover, different densities of the competitor and predatory fish (i.e. goldfish) allowed me to know the effect of their density on the coefficient of competition or predation.

Following Abramsky and Rosenzweig, ${ }^{30}$ drawing the slope of every isocline was conducted by using the following general formula:

$$
\alpha_{\text {guppy, gold }}=\frac{\Delta \text { guppy }}{\Delta \text { gold }}=\frac{\text { guppys }_{\text {left }}-\text { guppys }_{\text {right }}}{\text { gold }_{\text {left }}-\text { gold }_{\text {right }}}
$$

For all analyses, I defined the alpha value to be 0.05 and performed the analyses with SPSS version 16.

\section{Results}

As food pellet was equal in both sides of the experimental aquarium, time did not have significant effect $(\mathrm{df}=1 ; \mathrm{F}=1.06 ; \mathrm{P}=$ 0.86 ) on the distribution and abundance of guppies with the presence of goldfish as competitors or without competitors. This suggested that food does not have significant effect on the distribution and abundance of guppies, but the competitors (i.e. goldfish) significantly affected the distribution, abundance, and fitness of guppies in the experimental aquarium because goldfish seems to be territorial while acquiring food.

\section{Guppies' isodar}

The isodar was constructed by regressing the right side of guppies' density as an independent variable whereas the left side of the guppies' density as a dependent variable (Figure 1). The isodar equation obtained by regressing the right side versus the left side densities of guppies revealed that the intercept was not significant because it was less than 0. Moreover, the slope of the isodar was not significant because it was closer to 1 (Table 1), (Figure 1). This suggested that guppies had equal fitness in both sides of the experimental aquarium because the two sides of the aquarium are identical in terms of food availability and density of guppies.

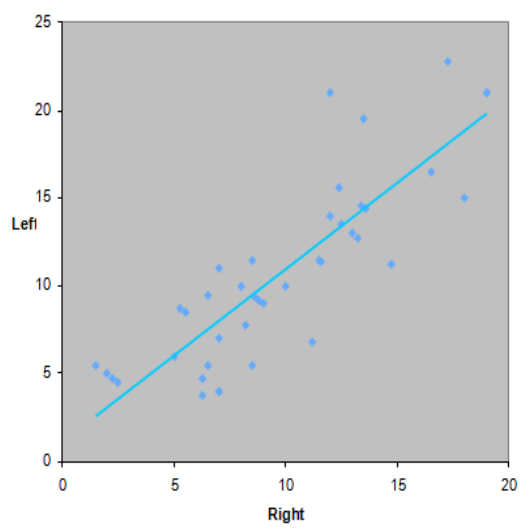

Figure I Isodar of guppies when they were alone and food pellets were provided in equal quantity in both sides of an experimental aquarium.

Table I The isodar equation obtained through the regression analysis of right versus left density of guppies

\begin{tabular}{lllll}
\hline Variable & Coefficients & P value & $\mathbf{- 9 5 . 0 0 \%}$ C.L & $\begin{array}{l}\mathbf{+ 9 5 . 0 0 \%} \\
\text { C.L }\end{array}$ \\
\hline Intercept & 1.179 & 0.278 & -0.963 & 3.322 \\
Right & 0.952 & 0.564 & 0.783 & 1.122 \\
\hline
\end{tabular}

\section{Isoclines and competition coefficients}

Since the population size was less than 30; therefore, a student- $t$ test was used to measure the competitive difference between the right versus left sides of the aquarium with and without competitors (i.e. goldfish). The result revealed that goldfish had a significant effect on the competition coefficient, suggesting that inter-specific competition significantly affected the distribution, abundance, and fitness of guppies in an experimental aquarium $(\mathrm{P}<0.001)$ Figure 2.

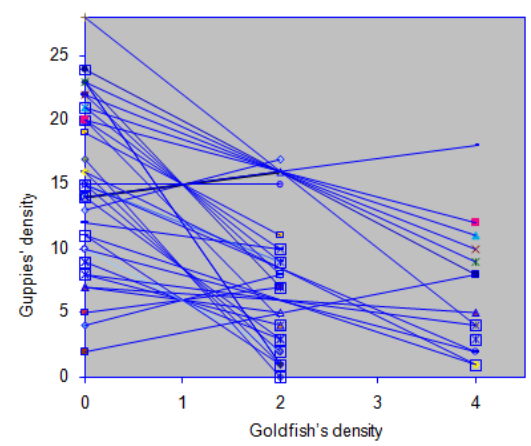

Figure $\mathbf{2}$ The isoclines of guppies where the density of goldfish is independent variable and regressed against the guppies' density as dependent variable. The isocline slope represents the competition coefficient. However, each line designates one isocline which was drawn by connecting two points that represent the densities of goldfish versus guppies in each side of the aquarium. 


\section{Effect of guppies' density on competition coefficient}

As the value of $\mathrm{P}=0.065$, the result of the simple linear regression revealed that guppies' density did not have a significant effect on the competition coefficient (Figure 3). Furthermore, polynomial regression was run to test the effect of guppies' density on competition coefficient (Table 2). The polynomial regression revealed that the density of guppies significantly affected the competition coefficient because the value of $\mathrm{P}<0.001$ (Table 2). Then, the following equation was developed from the result of the polynomial regression (Table 2) to explain how guppies' density affected the competition coefficient.

$$
\mathrm{Y}=17.47-2.15 \mathrm{X}+0.05 \mathrm{X} 2
$$

Where $\mathrm{Y}=$ Competition coefficient

\section{$\mathrm{X}=$ Guppies' density}

Now it is easy to find the minimum value for the competition coefficient using the above formula:

$$
\begin{aligned}
& Y^{\prime}=-2.15+0.1 X \\
& Y^{\prime}=-2.15+0.1 X=0
\end{aligned}
$$

Minimum point $\mathrm{X}=21.5 ; \mathrm{Y}=-5.71$

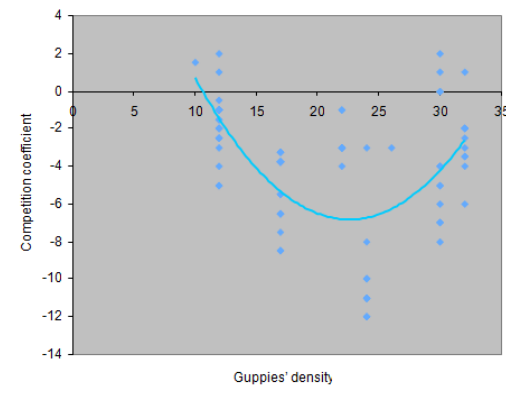

Figure 3 The total density of guppies in both sides of the aquarium was used as independent variable whereas competition coeficient was used as a dependent variable.

Table 2 Multiple polynomial regression results for competition coefficients measured against guppies' density $\left(X, X^{2}\right)$

\begin{tabular}{lllc}
\hline Variable & Coefficients & P value & Adjusted $\boldsymbol{R}^{\mathbf{2}}$ \\
\hline Intercept & 17.47 & $<0.00 \mathrm{I}$ & \\
Guppies & -2.15 & $<0.001$ & 0.39 \\
Guppies $^{2}$ & 0.05 & $<0.00 \mathrm{I}$ &
\end{tabular}

The effect of goldfish's density on competition coefficient

The result showed that the density of goldfish had a significant effect on the competition coefficient (Table 3), (Figure 4). However, the result of the multiple regression obtained by regressing the guppies' density versus goldfish's density did not significantly affect the competition coefficient because the value of $P=0.083$. Thus, the effect of goldfish's density on competition coefficient can be represented by simple linear regression equation using the following formula:

$\mathrm{Y}=-7.75+1.31 \mathrm{X}$

Where,

$\mathrm{Y}=$ Competition coefficient

$\mathrm{X}=$ Goldfish density

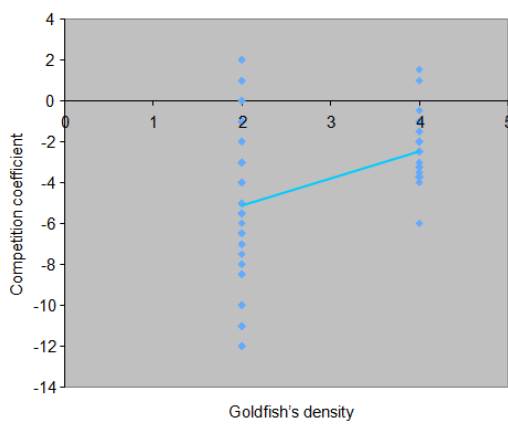

Figure 4 The density of goldfish was treated as independent variable whereas competition coefficient was regressed as a dependent variable.

Table 3 The separate effect of goldfish density on competition coefficient produced by employing simple regression technique

\begin{tabular}{lllc}
\hline Variable & $\begin{array}{l}\text { Competition } \\
\text { Coefficient }\end{array}$ & P value & Adjusted $\boldsymbol{R}^{\mathbf{2}}$ \\
\hline Goldfish (intercept) & -7.75 & $<0.001$ & 0.14 \\
Goldfish (slope) & 1.313 & $<0.001$ & \\
\hline
\end{tabular}

\section{Isoclines and predation coefficients}

A similar method was employed to draw the isoclines like what was used to draw the isoclines for the competition coefficient (Figure $5)$.

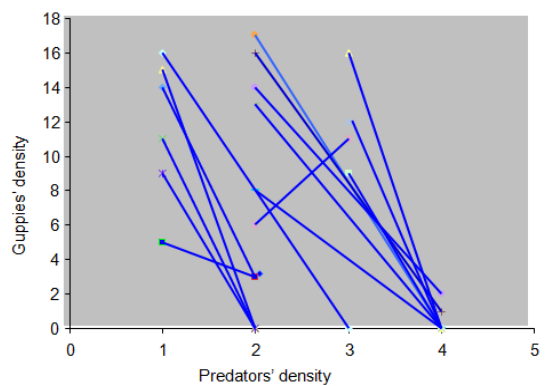

Figure $\mathbf{5}$ Isoclines of guppies where the density of the predator was treated as independent variable whereas the density of guppies as dependent variable. The Isocline slope represents the predation coefficient. Each line designates one isocline which was drawn by connecting two points that represent densities of predator versus guppies in each side of the aquarium.

\section{The effect of guppies' density on predation coefficient}

Using simple linear regression, the result showed that guppies' density did not show significant effect on predation coefficient because the value of $P=0.75$. Similarly, the result of the polynomial regression $(\mathrm{X}, \mathrm{X} 2)$ revealed that guppies' density did not have significant effect on predation coefficient because the value of $\mathrm{P}=0.170$ (Figure 6).

\section{The effect of predator's density on predation coefficient}

The result obtained by simple linear regression technique revealed that predator's density (i.e. goldfish as a predator) did not have a significant effect on predation coefficient because the value of $\mathrm{P}=$ 0.94. Furthermore, multiple polynomial regression technique was employed to detect the effect of predator's density on predation coefficient. Fortunately, the result of the multiple polynomial 
regression revealed that predator's density had a significant effect at (X, X2) including the intercept on the predation coefficient because the value of $\mathrm{P}<0.01$ (Table 4), (Figure 7).

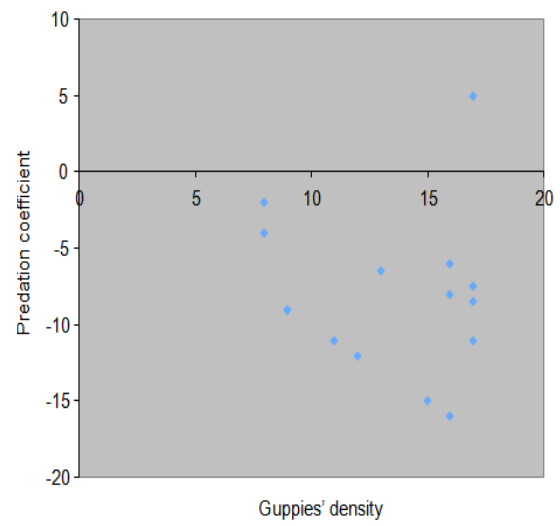

Figure 6 Guppies' density in both sides of the aquarium was treated as independent variable whereas predation coefficient was treated as dependent variable.

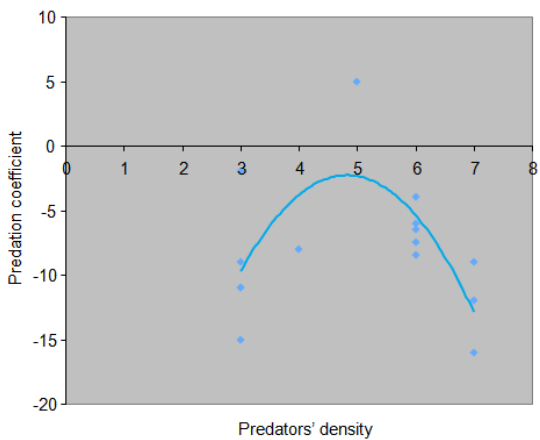

Figure 7 Predators' density in both sides of the aquarium was treated as independent variable whereas predation coefficient was assigned as dependent variable. This was done to test the effect of predator's density on predation coefficient.

Table 4 The effect of predator's density on predation coefficient produced by polynomial regression $\left(X, X^{2}\right)$

\begin{tabular}{lllc}
\hline Variable & Coefficients & P value & Adjusted $\boldsymbol{R}^{\mathbf{2}}$ \\
\hline Intercept & -54.486 & 0.004 & \\
Predator & 21.624 & 0.009 & 0.362 \\
$\mathrm{P}^{2}$ & -2.239 & 0.008 &
\end{tabular}

The equation for the coefficient of predation and the isocline could be developed for significant factors. In this case, only predation density had a significant effect on predation coefficient. Thus, the equation for the predation coefficient would be given by using:

$$
\mathrm{Y}=-54.486+21.624 \mathrm{X}-2.239 \mathrm{X} 2
$$

Where

$$
\begin{aligned}
& \mathrm{Y}=\text { Predation coefficient } \\
& \mathrm{X}=\text { Density of predator }
\end{aligned}
$$

As the slope of the isocline changed with density, obtaining the maximum value for the predation coefficient was important. This was obtained by using the following equation:

$$
\mathrm{Y}^{\prime}=21.624-4.486 \mathrm{X}
$$

\section{$21.624-4.486 \mathrm{X}=0$}

Maximum point would be when $\mathrm{X}=4.832$; and $\mathrm{Y}=-2.313$ (i.e. weakest predation coefficient)

Now it is easy to compute for the value of the isocline as followed:

$$
\begin{aligned}
& \mathrm{Y}^{\prime}=-54.486+21.624 \mathrm{X}-2.239 \mathrm{X} 2 \\
& \mathrm{Y}=\mathrm{C}-54.486 \mathrm{X}+21.624 \mathrm{X} 2 / 2-2.239 \mathrm{X} 3 / 3 \\
& \mathrm{Y}=\mathrm{C}-54.486 \mathrm{X}+10.811 \mathrm{X} 2-0.751 \mathrm{X} 3
\end{aligned}
$$

Thus, the value of $\mathrm{C}$ can be computed from the average values of the densities of guppies and predators as followed.

$$
\begin{aligned}
& \mathrm{Y}(\text { Guppies })=13.42, \mathrm{X}(\text { Predators })=5 \\
& \mathrm{C}=13.42+54.48 \times 5-10.81 \times 52+0.75 \times 53 \\
& \mathrm{C}=108.90
\end{aligned}
$$

Therefore, the isocline equation was given by:

$$
\mathrm{Y}=108.90-54.486 \mathrm{X}+10.811 \mathrm{X} 2-0.751 \mathrm{X} 3
$$

\section{Discussion}

Competition and predation are pervasive evolutionarily and ecological forces which play imperative role to determine the distribution, abundance, habitat selection, and population dynamics of different species in a biotic community, ${ }^{4,8,12-15}$ This study was conducted to test the effect of competition (i.e. intra-specific and inter-specific) and predation on the distribution, abundance, and fitness of guppies in an experimental aquarium under laboratory condition. The result showed that the movement of guppies in both sides of the aquarium was significantly affected by guppies' density, and the presence of goldfish as competitors and predators. Moreover, goldfish had territorial behavior while feeding ${ }^{54}$ so that their impact on the distribution, abundance, and fitness of guppies was strongly correlated with the effect of competition. Therefore, it is better to separately discuss the effect of each factor on the distribution, abundance, and fitness of guppies as followed.

\section{Effects of intra-specific competition}

The result of the simple linear regression revealed that guppies' density did not have a significant effect on the intra-specific competition coefficient (Figure 3). However, the result of the multiple polynomial regression (Table 2) showed that guppies' density had a significant effect on competition coefficient, suggesting that intraspecific competition was important factor in affecting the distribution, abundance, and fitness of guppies in an experimental aquarium under laboratory condition. Previous studies. ${ }^{5,30,55,56}$ also noted that intraspecific competition is important to affect the distribution, abundance, and fitness of desert rodents.

\section{Effects of inter-specific competition}

Previous field studies demonstrated that interference due to inter-specific competition had a significant effect on the distribution, abundance, and fitness of various species in a biotic community. $5,7,46,57,58$ The result of the simple linear regression revealed that the presence of goldfish as a competitor significantly affected $(\mathrm{P}<0.001)$ the interspecific competition coefficient, suggesting that the interference of goldfish significantly affected the distribution, abundance, and fitness of guppies (Table 3), (Figure 4). The territorial behavior of goldfish might contribute to affect the free movement of guppies in 
an experimental aquarium so that the density of goldfish may have a significant effect on the inter-specific competition coefficient. For example, Joseph ${ }^{54}$ noted that goldfish are naturally gregarious and also displaying territorial behavior while feeding. However, the effect of inter-specific competition due to the territorial behavior of the competitor (i.e. the goldfish) is beyond the scope of this study. Thus, it demands further studies just to investigate how the territorial behavior of goldfish will significantly contribute to affect the inter-specific competition coefficient and ultimately the distribution, abundance, and fitness of guppies.

However, the result obtained through the multiple polynomial regression revealed that the density of guppies significantly affected the inter-specific competition coefficient, suggesting that the effect of guppies density on the distribution, abundance, and fitness of goldfish was weaker than the counteract effect of the density of goldfish as a competitor on the distribution, abundance, and fitness of guppies. Similarly, in a field experiment conducted on two competitive desert rodent species in the Negev Desert of Israel, Abramsky et al., ${ }^{5}$ found that the density of Gerbillus pyramidum had a significant effect on the inter-specific competition coefficient, suggesting that interference of the dominant Gerbillus pyramidum significantly affected the distribution, abundance, and fitness of the co-dominant Gerbillus allenbyi. However, the density of Gerbillus allenbyi did not significantly affect the inter-specific competition coefficient, suggesting that Gerbillus allenbyi had weaker effect on the distribution, abundance, and fitness of Gerbillus pyramidum.

\section{Effects of predation}

Different ecological researchers in their classical theories noted that predation is one of the major and primary selective forces in nature which has been continuously shaping the distribution, abundance, habitat selection, population dynamics, behavior, and morphology of different prey species more significantly than the other natural selective forces do (e.g. competition). . $-5,7,15,44-46,57-59^{\text {The result of the }}$ multiple polynomial regression revealed that predation had significant effect on the predation coefficient (Figure 7), suggesting that presence of goldfish as a predator significantly affected the distribution, abundance, and fitness of guppies in an experimental aquarium. One of the possible reasons is that prey species are running for their lives, but predators are running for their dinner, which suggests that predators have stronger effect on their prey than preys do on their predators. This is because unlike competition, predation results in fatal accidents to prey species in a biotic community. However, predator density affected the predation coefficient in a non-linear fashion (Table 4), (Figure 7). Moreover, the result of this study revealed that predation coefficient had only stronger effect in high and low predator densities, but it had weak effect at the intermediate predator density (Figure 7). This result was in agreement with the results of many previous researches conducted on the distribution, abundance, and fitness of prey species..$^{3-5,7,46,57,58}$

\section{Conclusion}

Competition and predation are vital forces of evolution which allow species coexistence in biotic community where resources are limited in quantity and/or quality. The result of this study revealed that the distribution, abundance, and fitness of guppies' were affected by competition (i.e. intra-specific and inter-specific) and predation. The results of the coefficients of competition and predation suggested that the effect was density-dependent. Moreover, guppies' density affected the competition coefficient when goldfish were present as a competitor. However, guppies' density did not affect the predation coefficient when goldfish were present as predators, suggesting that guppies had weaker effect on the distribution, abundance, and fitness of their predators (i.e. goldfish). This is in contrast to the results of Abramsky et al., ${ }^{5}$ in which predation and inter-specific competition between two desert rodent species (i.e. Gerbillus allenbyi and Gerbillus allenbyi) was significant, while intra-specific competition was still important. The result of the present study would lead us to conclude that the presence of goldfish as predators more strongly affected the distribution, abundance, and fitness of guppies than did guppies as competitors on goldfish. This is supposed to be the effect of predation may even result in fatal accident which is very unlikely in the case of competition among individuals of different species in a biotic community. With regard to the comparison made between competition versus predation coefficients, it is difficult to decide which coefficient had stronger effect than the other. This is because both coefficients are affected by density as shown in the results of the polynomial regression. To conclude, if all parameters are kept equal, predation has the strongest effect on the distribution, abundance, and fitness of guppies followed by the effects of inter-specific and intraspecific competitions, respectively.

\section{Acknowledgement}

None.

\section{Conflict of interest}

Author declares that there is no conflict of interest.

\section{References}

1. Blanka DA, Ruckstuhld K, Yanga W. Development of juvenile goitered gazelle social behavior during the hiding period. Behav Processes. $2007 ; 144: 82-88$.

2. Ben-Natan G, Abramsky Z, Kotler BP, et al. Seeds redistribution in sand dunes: a basis for coexistence of two rodent species. Oikos. 2014;105:325-335.

3. Kotler BP, Ayal Y, Subach A. Effects of predatory risk and resource renewal on the timing of foraging activity in a gerbil community. Oecologia. 1994;100:391-396.

4. Kotler BP, Brown JS, Mitchell WA. The role of predation in shaping the behavior, morphology and community organization of desert rodents. Journal of Zoology. 1994;42:449-466.

5. Abramsky Z, Rosenzweig ML, Subach A. Do gerbils care more about competition or predation? Oikos. 1998;83:75-84.

6. Mac Arthur RH, Levins R. The limiting similarity, convergence, and divergence of coexisting species. The American Naturalist. 1967;101:377-385.

7. Abramsky Z, Strauss E, Subach A, et al. The effect of barn owls (Tyto $a l b a)$ on the activity and microhabitat selection of Gerbillus allenbyi and G. pyramidum. Oecologia. 1996;105: 313-319.

8. Ziv Y. The effect of habitat heterogeneity on species diversity patterns: a community-level approach using an object-oriented landscape simulation model (SHALOM). Ecological Modeling. 1998;111:135170 .

9. Rosenzweig ML. Optimal habitat selection in two species competitive systems. In: U Halbachand, J Jacobs, editors. Stuttgart: Population ecology, Gustav Fischer Verlag; 1979:283-293.

10. Rosenzweig ML. A theory of habitat selection. Ecology. 1981;62:327335 .

11. Rosenzweig ML. Hummingbird isolegs in an experimental system. Behavioral Ecology and Sociobiology. 1986;19:313-322. 
12. Abramsky Z, Rosenzweig ML, Brand S. Habitat selection in Israel desert rodents: comparison of a traditional and a new method of analysis. Oikos. 1985;45:79-88.

13. Brown JS. Patch use as an indicator of habitat preference, predation risk, and competition. Behavioral Ecology \& Sociobiology. 1988;22:37-47.

14. Brown JS. Patch use under predation risk: models and predictions. Ann Zool Fenn. 1992;29:301-309.

15. Brown JS. Vigilance, patch use, and habitat selection: foraging under predation risk. Evolutionary Ecology Research. 1999;1:49-71.

16. Brown JS, Mitchell WA. Diet selection on depletable resources. Oikos. 1989;54:33-43.

17. Jonzen N, Wilcox C, Possingham HP. Habitat selection and population regulation in temporally fluctuating environments. The American Naturalist. 2004;164(4):103-114.

18. Morris DW. Tests of density-dependent habitat selection in a patchy environment. Ecology Monographers. 1987;57(4):269-281.

19. Morris DW. Habitat dependent population regulation and community structure. Evolutionary Ecology Research. 1988;2:253-269.

20. Morris DW. State-dependent optimization of litter size. Oikos 1998;83:518-528.

21. Morris DW. How can we apply theories of habitat selection to wildlife conservation and management? Wildlife Research. 2003;30:303-319.

22. Krebs C. Niche measures and resource preferences. In: Ecological methodology. USA: Addison-Welsey Educational Publishers; 1999. 455-495.

23. Fretwell SD, Lucas HLJ. On territorial behavior and other factors influencing habitat distribution in birds. Acta Biotheoretica. 1969;19(1):16-36.

24. Fretwell SD, Lucas HLJ. On territorial behavior and other factors influencing habitat distribution in birds. Acta Biotheor. 1970;19:16-36.

25. Rosenzweig ML, Abramsky Z. Detecting density-dependent habita selection. The American Naturalist. 1985;126(3):405-417.

26. Cresswell W. Non-lethal effects of predation in birds. Ibis. 2008;150:317.

27. Abramsky Z, Rosenzweig ML, Subach A. Gerbils under threat of owl predation: isoclines and isodars. Oikos. 1997;78:81-90.

28. Lima SL, Dill LM. Behavioral decisions made under the risk of predation: A review and prospectus. Can J zool. 1990;68:619-640.

29. Crowell KL, Pimm SL. Competition and niche shift of mice introduced onto small islands. Oikos. 1976;27:251-258.

30. Abramsky Z, Rosenzweig ML. The shape of a gerbil isocline measured using principles of optimal habitat selection. Ecology. 1991;72:329 340 .

31. Vandermeer JH. Generalized models of two species interaction: a graphical analysis. Ecology. 1973;54: 809-818.

32. Brown JS, Kotler BP. Hazardous duty pay and the foraging cost of predation. Ecology Letters. 2004;7:999-1014.

33. Fortin D, Boyce MS, Merrill EH, et al. Foraging costs of vigilance in large mammalian herbivores. Oikos. 2004;107:172-180.

34. Heithaus MR, Wirsing AJ, Thomson JA, et al. A review of lethal and non-lethal effects of predators on adult marine turtles. Journal of Experimental Marine Biology \& Ecology. 2008;356:43-51.

35. Lima SL. Non-lethal effects in the ecology of predator-prey interactions BioScience. 1998;48:25-34.

36. McCauley SJ, Rowe L, Fortin MJ. The deadly effects of "non-lethal" predators. Ecology. 2011;92:2043-2048.

37. Luning J. Phenotypic plasticity of Daphnia pulex in the presence of invertebrate predators: morphological and life history responses. Oecologia. 1992;92:383-390.

38. Pangle KL, Peacor SD, Johannsson OE. Large non-lethal effects of an invasive invertebrate predator on zooplankton population growth rate. Ecology. 2007;88:402-412.

39. Wesner JS, Billman EJ, Belk MC. Multiple predators indirectly alter community assembly across ecological boundaries. Ecology. 2012;97:1674-1682.

40. McCollum SA, Leimberger JD. Predator-induced morphological changes in an amphibian: predation by dragonflies affects tadpole shape and color. Oecologia. 1997;109:615-621.

41. Preisser EL, Bolnick DI, Benard MF. Scared to death? The effects of intimidation and consumption in predator-prey interactions. Ecology. 2005;86:501-509.

42. Creel S, Christianson D. Relationships between direct predation and risk effects. Journal of Trends Ecol Evol. 2008;23:194-201.

43. Peacor SD, Werner EE. Context dependence of non-lethal effects of a predator on prey growth. Israel J Zool. 2004;50:139-167.

44. Kotler BP. Harvesting rates and predatory risk in desert rodents: a comparison of two communities on different continents. Mammalogy. 1984;65:91-96.

45. Kotler BP. Risk of predation and the structure of desert rodent communities. Ecology. 19984;65:689-701.

46. Kotler BP, Brown JS, Hasson O. Factors affecting gerbil foraging behavior and rates of owl predation. Ecology. 1991;72:2249-2260.

47. Magurran AE. Evolutionary Ecology: The Trinidadian Guppy. New York: Oxford University Press; 2005.

48. Froese R, Pauly D. Poecilia reticulata in FishBase. 2007.

49. Magurran AE, Phillip DAT. Evolutionary implications of large-scale patterns in the ecology of Trinidadian guppies, Poecilia reticulata. Biological Journal of the Linnean Society. 2001;73:1-9.

50. Dussault GV, Kramer DL. Food and feeding behavior of the guppy, Poecilia reticulata (Pisces: Poeciliidae). Canadian Journal of Zoology. 1981;59:684-701.

51. Seghers BH. Schooling Behavior in the Guppy (Poecilia reticulata): An Evolutionary Response to Predation. Evolution. 1974;28:486-489.

52. Richmond L. Goldfish (Carassius auratus). 2013.

53. Hugo M. The goldfish and its systematic culture with a view to profit 1883.

54. Joseph S. Goldfish varieties and genetics: A handbook for breeders. Oxford: Blackwell Science. 2001. 21 p.

55. Ovadia O, Abramsky Z. Density dependent habitat selection: evaluation of the Isodar method. Oikos. 1995;73:86-94.

56. Ziv Y, Abramsky Z, Kotler BP, et al. Interference competition and temporal and habitat partitioning in two gerbil species. Oikos. 1993;55:237-246.

57. Kotler BP, Brown JS, Smith RJ, et al. The effects of morphology and body size on rates of owl predation on desert rodents. Oikos. 1988 53:145-152.

58. Kotler BP, Blaustein L, Brown JS. Predator facilitation: the combined effect of snakes and owls on the foraging behavior of gerbils. Annales Zoology Fennici. 1992;29:199-206.

59. Kotler BP. Owl predation on desert rodents which differ in morphology and behavior. Mammalogy. 1985;66:824-828. 